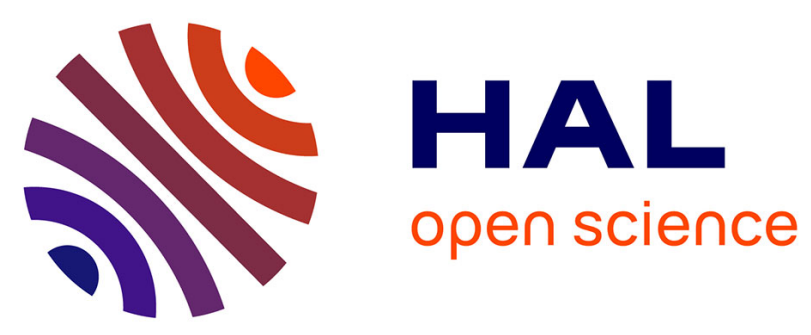

\title{
Low flux and low energy helium ion implantation into tungsten using a dedicated plasma source
}

\author{
Lucile Pentecoste, Anne-Lise Thomann, Amer Melhem, Amael Caillard, \\ Stéphane Cuynet, Thomas Lecas, Pascal Brault, Pierre Desgardin, \\ Marie-France Barthe
}

\section{To cite this version:}

Lucile Pentecoste, Anne-Lise Thomann, Amer Melhem, Amael Caillard, Stéphane Cuynet, et al.. Low flux and low energy helium ion implantation into tungsten using a dedicated plasma source. Nuclear Instruments and Methods in Physics Research Section B: Beam Interactions with Materials and Atoms, 2016, 383, pp.38-46. 10.1016/j.nimb.2016.06.011 . hal-01343361

\section{HAL Id: hal-01343361 \\ https://hal.science/hal-01343361}

Submitted on 8 Jul 2016

HAL is a multi-disciplinary open access archive for the deposit and dissemination of scientific research documents, whether they are published or not. The documents may come from teaching and research institutions in France or abroad, or from public or private research centers.
L'archive ouverte pluridisciplinaire HAL, est destinée au dépôt et à la diffusion de documents scientifiques de niveau recherche, publiés ou non, émanant des établissements d'enseignement et de recherche français ou étrangers, des laboratoires publics ou privés. 
Low flux and low energy helium ion implantation into tungsten using a dedicated plasma source

Lucile Pentecoste $^{1}$, Anne-Lise Thomann ${ }^{1}$, Amer Melhem ${ }^{1}$, Amael Caillard ${ }^{1}$, Stéphane Cuynet $^{1}$, Thomas Lecas ${ }^{1}$, Pascal Brault ${ }^{1}$, Pierre Desgardin ${ }^{2}$, Marie-France Barthe ${ }^{2}$

${ }^{1}$ GREMI, CNRS/Université d'Orléans, 14 rue d'Issoudun, B.P. 6744, 45067 Orléans Cedex2, France

${ }^{2}$ CNRS, UPR3079 CEMHTI, 1D avenue de la Recherche Scientifique, 45071 Orléans cedex2, France

E-mail : anne-lise.thomann@univ-orleans.fr

Keywords : ion implantation, ion energy distribution function, capacitively coupled discharge, helium implantation in tungsten, vacancy defect formation 


\begin{abstract}
The aim of this work is to investigate the first stages of defect formation in tungsten (W) due to the accumulation of helium $(\mathrm{He})$ atoms inside the crystal lattice. To reach the required implantation conditions, i.e. low He ion fluxes $\left(10^{11}-10^{14}\right.$ ions. $\left.\mathrm{cm}^{2} \cdot \mathrm{s}^{-1}\right)$ and kinetic energies below the $\mathrm{W}$ atom displacement threshold (about $500 \mathrm{eV}$ for $\mathrm{He}+$ ), an ICP source has been designed and connected to a diffusion chamber. Implantation conditions have been characterized by means of complementary diagnostics modified for measurements in this very low density helium plasma. It was shown that lowest ion fluxes could only be reached for the discharge working in capacitive mode either in $\alpha$ or $\gamma$ regime. Special attention was paid to control the energy gained by the ions by acceleration through the sheath at the direct current biased substrate. At very low helium pressure, in $\alpha$ regime, a broad ion energy distribution function was evidenced, whereas a peak centered on the potential difference between the plasma and the biased substrate was found at higher pressures in the $\gamma$ mode. Polycrystalline tungsten samples were exposed to the helium plasma in both regimes of the discharge and characterized by positron annihilation spectroscopy in order to detect the formed vacancy defects. It was found that $\mathrm{W}$ vacancies are able to be formed just by helium accumulation and that the same final implanted state is reached, whatever the operating mode of the capacitive discharge.
\end{abstract}




\section{Introduction}

Helium ion/tungsten (W) interaction has widely been studied in the past, especially in research works conducted in the frame of the International Thermonuclear Experimental Reactor (ITER). For its intrinsic properties, tungsten (W) was chosen to face the fusion plasma and thus will be submitted to high thermal and particle (neutrons, He+ etc.) fluxes [1$3]$. It has been shown that under corresponding ion fluxes, $\mathrm{He} / \mathrm{W}$ interaction could lead to the formation of holes, blisters, dusts and even of porous structures at the metal surface called fuzz [4-6]. These last fiber-form nanostructures could modify the surface properties (optical, wettability, specific surface etc.) of metals in a favorable way for various applications like catalysis for instance. Appearance of these porous structures has been reported for high ion fluxes and at low kinetic energies, indicating that defects responsible for the surface modification are due to the accumulation of helium in the metal lattice, rather than to the direct impact of ions on tungsten atoms.

To investigate and, in the case of ITER to monitor the formation of such structures, the study of the first steps of the involved elementary mechanisms is necessary. We thus investigated the implantation of $\mathrm{He}+$ at low fluxes and kinetic energies lower than $503 \mathrm{eV}$, which is the displacement threshold of $\mathrm{W}$ atoms by helium ions [7]. Under these conditions, the formation of vacancies by momentum transfer could be avoided, and the influence of He accumulation inside the $\mathrm{W}$ lattice could be evidenced.

Experimentally, we chose to perform implantations in a plasma environment, which means using a home-made plasma implantation source. Previous studies have shown the advantages of using a DC biased substrate holder inside a radio-frequency inductively coupled plasma (RF-ICP) source [8,9]. In such a configuration the ion energy, driven by the bias voltage, and the ion flux, related to the plasma density, could be separately controlled. However, limitations arise when very low ion fluxes are required. To reach a wide range of fluxes, from $10^{11}$ ions. $\mathrm{cm}^{-2} \mathrm{~s}^{-1}$ to $10^{14}$ ions. $\mathrm{cm}^{-2} \mathrm{~s}^{-1}$, an ICP-source connected to a diffusion chamber was designed. First, the source geometry and features were investigated, second the low density He plasma was investigated at the substrate position by means of a Langmuir probe, a retarding field analyzer (RFA [10]), an energy flux diagnostic [11,12] and, third, typical implantation conditions were defined. Finally, polycrystalline W substrates were implanted by He ions in selected conditions and analyzed by means of positron annihilation spectroscopy (PAS) to investigate the formation of vacancy defects. The implanted helium amount was determined by nuclear reaction analysis (NRA).

\section{Experimental details}

An experimental set up composed of a helium ICP source has been designed in order to control the ion flux on the substrate surface on a large scale. It is composed of an ICP source, made of a borosilicate glass tube (length $250 \mathrm{~mm}$, inner diameter $50 \mathrm{~mm}$ ) surrounded by a copper-coil antenna (number of turns is to 1 or 4 ) where the RF current flows. The source is connected to a stainless steel diffusion chamber (see figure 1) and the extremity of the tube, where the He gas is introduced, is grounded. The RF power supply used is a Dressler Cesar $1310(13.56 \mathrm{MHz}, 1000 \mathrm{~W})$ connected to a matching network Dressler $(13.56 \mathrm{MHz}, 1000 \mathrm{~W})$. A DC polarizable substrate holder (voltage-supply: Midec SK 600V-0,5A max) is introduced in front of the plasma source inside the diffusion chamber. The whole vessel is evacuated 
down to $10^{-7}$ mbar by a dry primary vacuum pump (ACP15 from Adixen) and an ATP150 (Adixen) turbomolecular pump. The distance between the substrate holder and the copper antenna can be varied from 15 to $65 \mathrm{~cm}$. A resistance is placed between the DC generator and the substrate holder in order to measure the ionic current and thus, deduce the ion flux incoming at the substrate surface. Since the secondary electron emission coefficient on tungsten for $300 \mathrm{eV}$ helium ions is expected to be about 0.24 [13], the ion flux value is taken $81 \%$ of the measured current.

Langmuir probe measurements were carried out at the substrate location (the substrate holder being removed from the chamber) with a SMARTPROBE from Scientific System, equipped with a passive compensation circuit. Typical distance between the probe and the antenna was $35 \mathrm{~cm}$. Because the He plasma is very tenuous at the substrate location, the collecting surface of the commercial Langmuir probe (initially tungsten wire) was replaced by a copper plate of $1 \mathrm{~cm}$ in diameter. This disc was placed perpendicularly to the diffusing plasma and its backside was covered by an insulating paste so that only the surface facing the plasma collects the ion current. Even with this extended collecting surface, signals were still very low and noisy and all I (V) curves were averaged over 5 to 10 sweeps and properly smoothed.

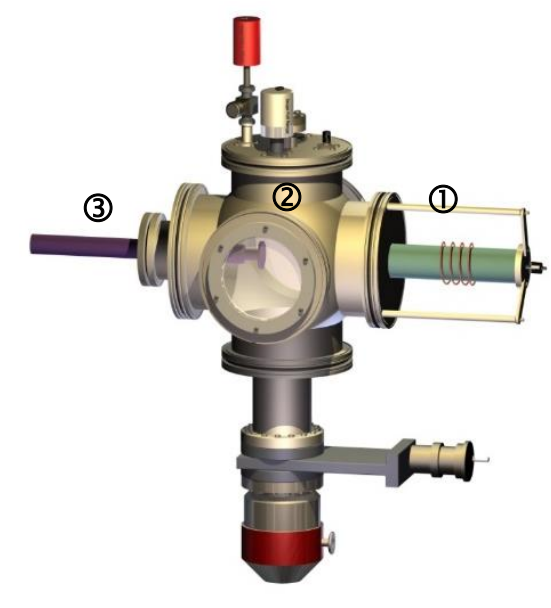

Fig. 1. Schematic of the experimental setup: 1) the RF-ICP source connected to 2) the diffusion chamber equipped with 3) a polarizable substrate holder; 4) the vacuum pump.

To evidence the ion energy distribution function (IEDF) a simplified retarding field analyzer was designed (see figure 2). A stainless steel grid located $0.9 \mathrm{~mm}$ from the substrate holder is biased at the desired voltage $\mathrm{V}_{\mathrm{b}}$. For those measurements the substrate holder is used as the collecting and discriminating device, its bias is varied from $\mathrm{V}_{\mathrm{b}}$ to $60 \mathrm{~V}$. In this work $\mathrm{V}_{\mathrm{b}}$ values were $-300 \mathrm{~V}$ and $-500 \mathrm{~V}$. The short distance between the biased grid and the substrate holder ensures that no collisions (elastic nor inelastic) between ions and neutrals will take place at that location, thus the IEDF resulting from the acceleration through the sheath formed between the plasma and the grid (biased at $\mathrm{V}_{\mathrm{b}}$ ) will not be modified during the measurements. 


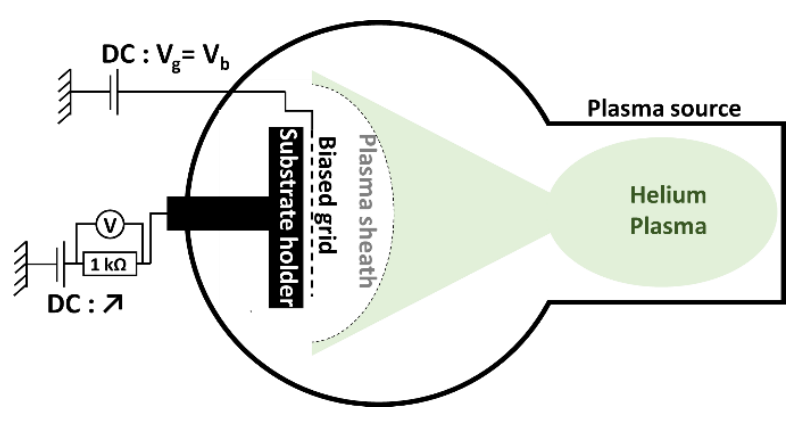

Fig. 2. Schematic of the system used to determine the IEDF: The stainless steel grid is biased at a given voltage $\mathrm{V}_{\mathrm{b}}$ and the ionic current is collected at the substrate holder $(50 \mathrm{~mm}$ in diameter) for varying bias voltages.

The current measured on the substrate holder (acting as a collector) is recorded with respect to the discriminating voltage, i.e. the kinetic energy of the collected ions. The corresponding distribution functions (that means the probability that an ion carrying a given kinetic energy reaches the surface) are determined from the derivative of these curves [14]. In the present configuration secondary electrons are not removed from the measured current, consequently the obtained curves are a convolution between the true IEDF and the electron secondary emission probability depending on the ion kinetic energy. Since we do not know the evolution of this probability in the investigated energy range, only qualitative comparison can be made from a plasma condition to another at a given $V_{b}$ value. This configuration (biased grid, variation of $\mathrm{V}_{\mathrm{b}}$ ) is only used for IEDF measurements. The grid is removed for implantation experiments.

In addition to the determination of the $\mathrm{He}^{+}$energy distribution, we have investigated the energy actually deposited by the helium plasma on the substrate by carrying out energy flux measurements with a diagnostic designed in GREMI laboratory. A detailed description of this tool could be found in previous works $[11,12]$. It is composed of a thermopile which enables to detect all kinds of energetic contributions (radiative, momentum transfer, chemical reaction etc.) with a sensitivity less than $1 \mathrm{~mW} . \mathrm{cm}^{-2}$. The active surface of the sensor measures $6 \mathrm{~mm}$ in diameter. It is inserted inside a copper rod of quite the same size than the substrate holder, thus local modification of the He plasma is expected to be similar as compared to implantation experiments. To perform measurements on a biased surface a copper chip $(6 \mathrm{~mm}$ diameter) is glued on the sensor with a thermal paste. This chip is connected to a DC voltage generator. As for the substrate holder, a resistance can be added to the circuit to record the ion current during the energy flux measurements. The bias voltage can be varied in the range $500 \mathrm{~V}$ to $0 \mathrm{~V}$.

Samples for implantation are $300 \mu \mathrm{m}$ thick polycrystalline $\mathrm{W}$ foils of $7 \times 7 \mathrm{~mm}^{2}$. Before analysis $\mathrm{W}$ samples are polished and annealed to remove as much as possible initial defects. The preparation of the tungsten substrates consists in 3 steps of scraping with sandpaper of various grain size $\left(300,600,1200 \mathrm{p} / \mathrm{cm}^{-2}, 15\right.$ to 20 minutes each) and 4 steps of polishing with polishing carpets covered with diamond paste with decreasing particle size $(6,3,1,1 / 10$ 
$\mu \mathrm{m}, 15$ to 20 minutes each). Those steps allow obtaining a mirror polished surface ready to be annealed in vacuum for an hour at $1600^{\circ} \mathrm{C}$.

Two samples are mechanically fixed on a $50 \mathrm{~mm}$ in diameter substrate holder especially designed in $\mathrm{W}$ to avoid contamination by another chemical element ( $\mathrm{Al}, \mathrm{Fe}$ etc.) that could be sputtered away from the holder under $\mathrm{He}^{+}$bombardment and re-deposited on the surface during implantation. All surface analyses were carried out ex-situ after sample storage in air. Typical delay between implantation experiments and analyses was 1 to 2 months.

Doppler broadening-positron annihilation spectroscopy (DB-PAS) has been performed at the CEMHTI laboratory on several $\mathrm{W}$ polycrystalline samples before and after implantation. This method allows to identify and quantify the vacancy defects in materials. It is based on the study of the shape of the $\gamma$ ray signal emitted during the annihilation mechanism. A detailed description of the apparatus and the basics of this technique can be found in references [8, 15]. In this work the $S$ parameter will be followed in function of the positron energy, especially in the 0-5 keV range, to diagnose the extreme surface of $\mathrm{W}$ samples. The $\mathrm{S}$ shape parameter of the $\gamma$ ray signal corresponds to the positrons which have experienced annihilation with electrons of low kinetic moment, i.e. valence electrons. Variations of this parameter in $\mathrm{W}$ for various densities and natures of vacancies have been investigated and are well known [16,17]. Results obtained in the present work will be compared to specific studies on DB-PAS characterization of $\mathrm{W}$ [8].

Nuclear reaction analysis (NRA) technique has been used to quantify the He implanted amount in $\mathrm{W}$. This has been done by performing implantation with ${ }^{3} \mathrm{He}$ isotope and bombarding the implanted material with $900 \mathrm{keV}$ deuterium beam which induces the reaction: ${ }^{3} \mathrm{He}\left(\mathrm{d},{ }^{1} \mathrm{H}\right) \propto[18-20]$. Protons are detected and the intensity of the corresponding signal is compared to that obtained on a reference sample containing $10^{16} \mathrm{He} \cdot \mathrm{cm}^{-2}$. This analysis has been carried out in a dedicated apparatus DIADDHEM at the CEMHTI laboratory, Orléans [18].

\section{Results and discussion}

\subsection{Plasma source features}

First of all the breakdown threshold of the He discharge has been studied for various positions of the antenna along the glass tube and for different numbers of coil turns. The evolution of the breakdown power with respect of the antenna position (not shown here) looks like the overlapping of two Paschen curves [21-23]. The discharge breakdown threshold depends on the distance between the antenna and the glass tube sides, which are grounded. This was expected since at low power, ICP plasmas are known to operate in capacitive mode, characterized by low electron density and faint light emission. The features of such mode depend on the distance between the powered electrode (the antenna) and the earth plane [24]. In a large range of antenna positions the discharge is easy to ignite and the breakdown power is low, around $10 \mathrm{~W}$. In the following the antenna will be set in the middle of the glass tube. 
As mentioned above, ICP discharges could operate in a low density capacitive regime. Even in the so-called inductive regime a part of the coupling remains capacitive, unless a Faraday shield is added to the experimental device [24-26], which is not the case in the present work. Transition from capacitive coupling (CC) to inductive coupling (IC) (or E to H mode) depends on the gas pressure and on the applied RF power.

Evolution of the ion flux measured on the substrate holder with respect to the pressure in helium gas is given in figure 3a with a 4 turns coil antenna and at 50W. Based on the ionic current values and on the shape and localization of luminous zones, two different regions can be defined on this graph.

In region (1) at low pressures, the ion flux is very low and the plasma light emission almost undetectable. At $7 \mu$ bar, the ion flux sharply increases and the discharge is now visible inside the glass tube, located between the antenna and the two grounded sides (see figure $3 \mathrm{~b}$ ). A bright zone also exits inside the chamber, showing that the ionization certainly occurs in a large volume. The features of the discharge in this region indicate thus that the main coupling is capacitive. The sharp rise at $7 \mu$ bar could correspond to the transition between well-known $\alpha$ and $\gamma$ modes of the capacitive regime [27, 28].

At $14 \mu \mathrm{bar}$, at the frontier between region (1) and (2) on the graph, a peak is detected in the ion flux evolution and the light emission inside the chamber evolves. It turns from bright light to dark pink, whereas the discharge between the antenna and the grounded sides of the glass tube is enhanced. These changes, associated to a clear trend of the ion flux to decrease as the pressure increases from this step, shows that the plasma is now mainly created inside the glass tube and that ions reach the substrate holder after diffusion through the chamber. The decrease of the ions flux with increasing pressure is due the scattering effect caused by collisions between neutrals and ions created close to the antenna. The discharge, exhibiting an inductive part, becomes denser and denser inside the tube as the pressure is increased from $18 \mu$ bar to $30 \mu$ bar. From this pressure a very luminous plasma is visible well confined at the antenna position, which only weakly diffuses (see figure $3 \mathrm{~b}, 50 \mu \mathrm{bar}$ ): the discharge mainly operates in the inductive regime.

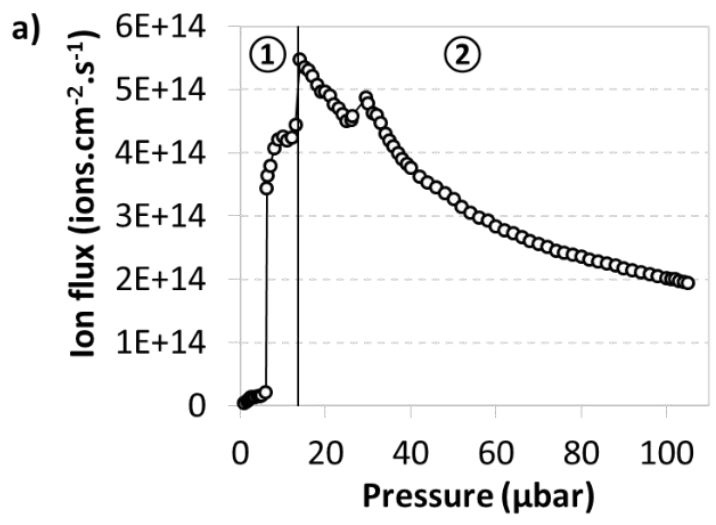

b)
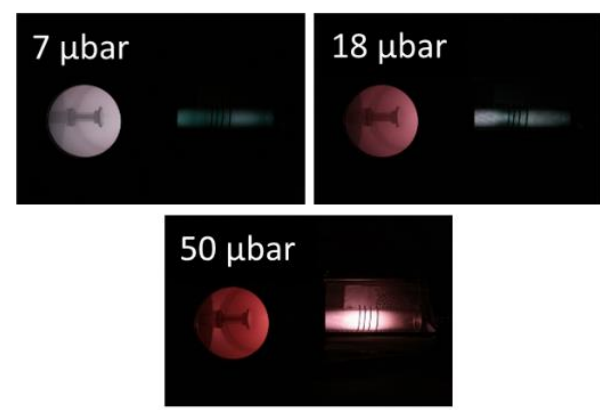

Fig. 3. a) Ion flux measured on the substrate holder biased at $-300 \mathrm{~V}$ in helium plasma, $35 \mathrm{~cm}$ away from the antenna and for an input RF power of $50 \mathrm{~W}$. Two operating regions are evidenced; b) appearance of the discharge for three pressures belonging to the regions defined 
in figure 3a: $7 \mu$ bar region (1) just after the sharp increase of the ion flux, $18 \mu$ bar and $50 \mu \mathrm{bar}$ region (2). On the pictures, the substrate holder inside the diffusion chamber is visible through circular window at left, and the plasma source (glass tube surrounded by the copper coil antenna) at right.

Evolution of the ion flux with respect to the RF power is given in figure 4 for five pressures belonging to both regions defined in figure 3 .

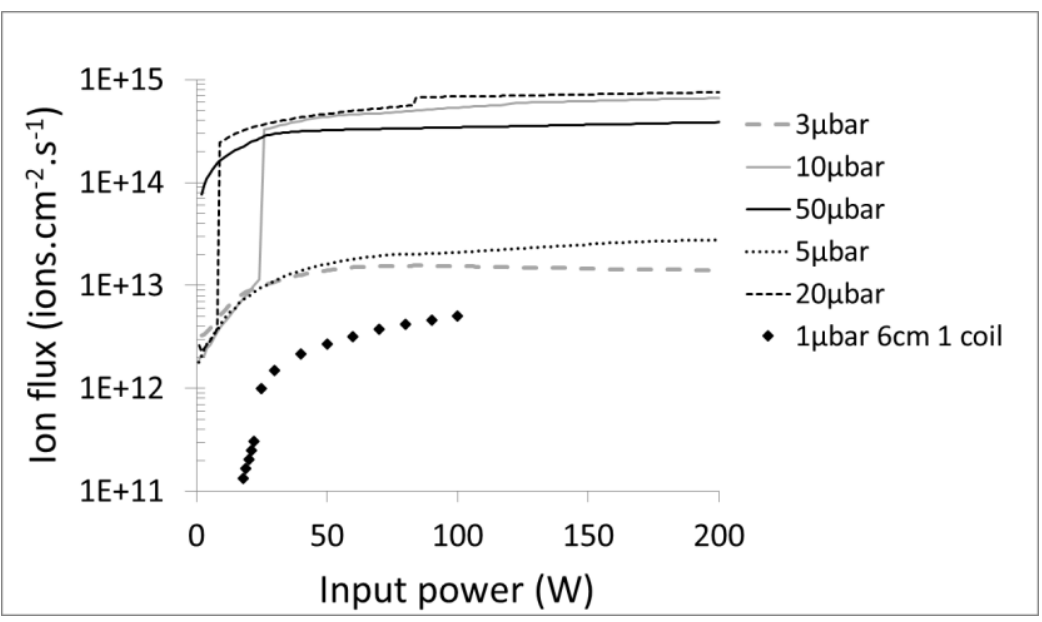

Fig. 4. Evolution of the ion flux with respect of the RF input power applied to the copper antenna for five helium pressures. When not given the substrate-antenna distance is $35 \mathrm{~cm}$ and the number of coils is 4 .

At 3, 5 and $50 \mu$ bar, which means in $\alpha-C C$ or IC regimes, respectively, the ion flux exhibits the same behavior: a sharp increase until $20 \mathrm{~W}-30 \mathrm{~W}$, followed by a steady state. But the ion flux value is more than one order of magnitude higher at $50 \mu \mathrm{bar}$ in the IC regime. At $10 \mu \mathrm{bar}$ a clear jump is observed. The ion flux abruptly turns from $10^{13}$ to $10^{14}$ ions. $\mathrm{cm}^{-2} \mathrm{~s}^{-1}$ at around $25 \mathrm{~W}$. This behavior suggests that a transition from $\alpha$ to $\gamma$ regimes takes place, which can occur for either increasing pressures or powers [28].

From curves presented in figure $3 \mathrm{a}$ and figure 4 it is seen that the ion flux order of magnitude is closely related to the discharge coupling. This was expected since coupling determines the bulk electron (ion) density, but also plays a role on the plasma confinement/diffusion in the chamber. For instance it has been shown by Peres et al [24] that CC produces less confined plasmas. This is in agreement with the plasma visible emission shown on figure $3 \mathrm{~b}$, which is the highest in the diffusion chamber. The trend is reverse in region (2) for IC, where the plasma seems to be well confined inside the tube. When the CC can be ignored, Peres et al showed that the confinement is sensitive to the coil configuration and to the dimensions of both the plasma source and the diffusion chamber [24]. 
To sum up, working in the $\alpha-C C$ will allow to reach fluxes in the range $2 \times 10^{12}$ to $3 \times 10^{13}$ ion. $\mathrm{cm}^{-2} \mathrm{~s}^{-1}$; highest values from $2 \times 10^{14}$ to $7 \times 10^{14}$ ion. $\mathrm{cm}^{-2} \mathrm{~s}^{-1}$ could be obtained both with $\gamma$ $\mathrm{CC}$ or IC discharges. Intermediate ions fluxes $\left(3 \times 10^{13}\right.$ to $10^{14}$ ion. $\left.\mathrm{cm}^{-2} \mathrm{~s}^{-1}\right)$ are not accessible in $\mathrm{CC}$ because of the $\alpha$ to $\gamma$ jump, but can be obtained in the IC mode by moving away the substrate holder from the source (not shown here). For the lowest required values $\left(10^{11}\right.$ ion. $\mathrm{cm}^{-2} \mathrm{~s}^{-1}$ ) the geometry of the antenna has to be modified. The evolution of the ion flux with the input RF power for a single turn coil antenna at $1 \mu \mathrm{bar}$ and $6 \mathrm{~cm}$ is also displayed in figure 4. It is clearly shown that these values of the ion flux can be also reached, and the wide range $10^{11}$ to $10^{14}$ ions.cm $\mathrm{cm}^{-2}$ can be achieved with the designed source.

However, in addition to a given and well controlled flux, other requirements have to be fulfilled to perform accurate ion implantations: 1) no or few collisions have to take place between ions and neutrals in the accelerating sheath between the plasma and the biased sample so that the ion energy distribution function would be narrow and centered on the potential difference between the plasma and the biased substrate; 2) only the ions bombarding the substrate should transfer energy. That means the plasma source should not increase in temperature (IR emission) when operating, in order to avoid undesired substrate heating phenomenon. To check these points, the He plasma has been characterized at typical sample positions.

\subsection{Characterization of the He diffusion plasma}

A complete investigation of the discharge regimes is out of the scope of the present work. We only aim at characterizing the plasma at the substrate location and finding out stable conditions of He implantation. Moreover in the present conditions (low mass gas, RF power, low density plasma), it is known that the accuracy of Langmuir probe measurements could be questionable. The RF compensation system is not completely suitable, which could prevent from safely treatment of the electronic part. Moreover in CC regime fluctuation of the plasma potential could disturb the $\mathrm{I}(\mathrm{V})$ characteristics.

Nevertheless, think that the order of magnitude of plasma parameters that are roughly determined from these measurements allows to evaluate whether the sheath formed at the biased substrate is collisionless or not, which is the main goal.

We chose to work in the range of power $50 \mathrm{~W}-100 \mathrm{~W}$ and of pressure $1 \mu \mathrm{bar}-10 \mu$ bar where $\alpha-\mathrm{CC}$ or $\gamma-\mathrm{CC}$ regimes could be established. The plasma potential $\left(\mathrm{V}_{\mathrm{p}}\right)$ and the floating potentials $\left(\mathrm{V}_{\mathrm{f}}\right)$ were determined on the $\mathrm{I}(\mathrm{V})$ curves obtained with the Langmuir probe $(\mathrm{I} "(\mathrm{~V})=0$ and $\mathrm{I}(\mathrm{V})=0$ respectively). The electron temperature $(\mathrm{kTe})$, was calculated using the relation [29]:

$$
V_{f}-V p=\frac{1}{2} \frac{k_{B} T_{e}}{e} \ln \left(\frac{1.571 m_{e}}{m_{i}}\right)
$$

Were $\mathrm{k}_{\mathrm{B}}$ is the Boltzmann constant $\left(1.38 \times 10^{-23} \mathrm{~m}^{2} \cdot \mathrm{kg} \cdot \mathrm{s}^{-2} \cdot \mathrm{K}^{-1}\right), \mathrm{m}_{\mathrm{e}}$ and $\mathrm{m}_{\mathrm{i}}$ the electron and ion mass, respectively. The electron density, $\mathrm{N}_{\mathrm{e}}$ supposed to equal the ion density was estimated 
from the ionic saturation part $\left(\mathrm{I}_{\mathrm{si}}\right.$ at $\left.-80 \mathrm{~V}\right)$ of the $\mathrm{I}(\mathrm{V})$ curve, where RF perturbations are expected to be the most limited [30]:

$$
I_{s i}=0.6 N_{e} e C_{s} A_{p}
$$

Where e is the electron charge, $\mathrm{C}_{\mathrm{s}}$ the Bohm velocity given by:

$C_{S}=\sqrt{\frac{k_{B} T_{e}}{m_{i}}}$

and $\mathrm{A}_{\mathrm{p}}$ being the probe size: $0.785 \mathrm{~cm}^{2}$

The above equation for $\mathrm{I}_{\mathrm{si}}$, and thus $\mathrm{A}_{\mathrm{p}}$ as the collecting surface, were used because most of the I(V) curves exhibit an ionic part that tends to saturate or only slightly evolves.

Results are presented in table 1.

\begin{tabular}{|c|c|c|c|c|c|}
\hline Pressure $(\mu$ bar $)$ & Power(W) & $\mathrm{V}_{\mathrm{p}}(\mathrm{V})$ & $\mathrm{V}_{\mathrm{f}}(\mathrm{V})$ & $\begin{array}{l}\mathrm{kT}_{\mathrm{e}} \\
(\mathrm{eV})\end{array}$ & $\mathrm{N}_{\mathrm{e}}(\mathrm{cm}-3)$ \\
\hline $\begin{array}{c}3 \\
\text { Region (1 }\end{array}$ & 50 & 18.6 & 12.7 & 1.4 & $7.3 * 10 \mathrm{E} 6$ \\
\hline \multirow{2}{*}{\begin{tabular}{c} 
Region (2) \\
\cline { 2 - 6 }
\end{tabular}} & 100 & 19.2 & 16.2 & 0.7 & $3.210 \mathrm{E} 7$ \\
\hline $\begin{array}{c}10 \\
\text { Region (2) }\end{array}$ & 50 & 39.5 & 18.5 & 4.8 & $8.2 * 10 \mathrm{E} 7$ \\
\cline { 2 - 6 } & 100 & 41.3 & 19.1 & 5.1 & $1.0 * 10 \mathrm{E} 8$ \\
\hline
\end{tabular}

Table 1. Helium plasma parameters determined from Langmuir probe measurements. $V_{p}$ and $V_{f}$ were evaluated on the $I(V)$ curves $\left(I \prime(V)=0\right.$ and $I(V)=0$ respectively), $\mathrm{kT}_{\mathrm{e}}$ was calculated with the Laframboise formula and $\mathrm{N}_{\mathrm{e}}$ from the ionic saturation part of the $\mathrm{I}(\mathrm{V})$ curve.

It appears from these data that the He plasma exhibits different features at low pressures (below $7 \mu$ bar) and high pressures (above $7 \mu$ bar). At helium pressures below $7 \mu$ bar, electron temperature and density are low: of the order of $1 \mathrm{eV}$ and of $10^{7} \mathrm{~cm}^{-3}$, respectively. For higher pressures the electron temperature increases up to about $6 \mathrm{eV}$, the electron density lies in the range $10^{8} \mathrm{~cm}^{-3}$. This corresponds to the transition evidenced in the region (1) of the ion flux evolution versus the pressure (figure $3 \mathrm{a}$ ).

An increase of the plasma potential, as observed in the present work as the pressure increases, is not expected when the discharge coupling turns from capacitive to inductive. On the contrary a high plasma potential is related to the existence of capacitive heating of the electrons [27]. This confirms, thus, that the discharge keeps a strong CC nature in this pressure range. The observed increase of the electron density could highlight the $\alpha$ to $\gamma$ regime transition in the $\mathrm{CC}$ mode. But this is usually associated with a decrease of $\mathrm{kT}_{\mathrm{e}}$. However, Seo et al reported that during transition from $\mathrm{CC}$ to IC mode an intermediate state is reached with high plasma potential and enhanced $\mathrm{N}_{\mathrm{e}}$ and $\mathrm{kT}_{\mathrm{e}}$, as compared to the pristine $\mathrm{CC}$ regime [31]. This is similar to the present observations and supports the idea that in the high pressure part of region (1) the discharge still operates in CC regime. 
To estimate the collision number into the accelerating sheath for a bias voltage of $-300 \mathrm{~V}$, the Debye length $\left(\lambda_{D}\right)$ and the sheath width $\left(L_{S}\right)$ were evaluated from the following formulae [29]:

$$
\begin{gathered}
\lambda_{D}^{2}=\frac{\varepsilon_{0} k_{B} T_{e}}{N_{e} e^{2}} \\
L_{S}=\lambda_{D}\left(\frac{e\left(V_{b}-V_{p}\right)}{k_{B} T_{e}}\right)^{\frac{3}{4}}
\end{gathered}
$$

They are compared to the mean free path of helium ions for elastic and inelastic collisions ( $\left.\lambda_{\text {IMFP }}, \lambda_{\text {EMFP }}\right)$ at the corresponding pressure.

Elastic mean free path is determined from the gas theory:

$$
\lambda_{E M F P}=\frac{1}{N \sigma}
$$

With $\mathrm{N}$ the neutral density, $\sigma=\pi \times\left(r_{\mathrm{He}+}+r_{\mathrm{He}}\right)^{2}$ the cross section, and, $r_{\mathrm{He}+}=$ $0.265 \AA$ and $r_{H e}=0.49 \AA$ being the helium ion and neutral radius, respectively.

Inelastic process that helium ions could undergo in the accelerating sheath is a charge transfer, which produces a fast neutral and an ion at rest. The cross section of such a mechanism has been estimated for helium by many authors to $10^{-15} \mathrm{~cm}^{2}$ at low kinetic energy [32-35]. $\lambda_{\text {IMFP }}$,

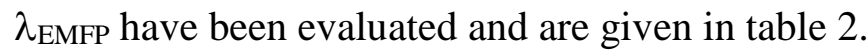

\begin{tabular}{|c|c|c|c|c|c|}
\hline Pressure $(\mu$ bar $)$ & Power $(\mathrm{W})$ & $\lambda_{\mathrm{D}}(\mathrm{cm})$ & $\mathrm{L}_{\mathrm{s}}(\mathrm{cm})$ & $\begin{array}{l}\lambda_{\text {EMFP }} \\
(\mathrm{cm})\end{array}$ & $\begin{array}{l}\lambda_{\text {IMFP }} \\
(\mathrm{cm})\end{array}$ \\
\hline \multirow{2}{*}{3} & 50 & 0.32 & 17 & 76 & 10.6 \\
\cline { 2 - 5 } & 100 & 0.11 & 10 & & \\
\hline \multirow{2}{*}{7} & 50 & 0.18 & 4 & 33 & 4.6 \\
\cline { 2 - 5 } & 100 & 0.17 & 3 & & \\
\hline \multirow{2}{*}{10} & 50 & 0.19 & 3 & 23 & 3.2 \\
\cline { 2 - 5 } & 100 & 0.22 & 3 & & 11 \\
\hline 20 & -- & -- & -- & 5 & 0.6 \\
\hline 50 & -- & -- & -- & & \\
\hline
\end{tabular}

Table 2. Debye length $\left(\lambda_{\mathrm{D}}\right)$, sheath width $\left(\mathrm{LS}_{\mathrm{S}}\right)$ and helium ion mean free path for elastic collisions $\left(\lambda_{\text {EMFP }}\right)$ and charge transfer $\left(\lambda_{\text {IMFP }}\right)$ for various plasma conditions at a biased voltage of $300 \mathrm{~V}$. 
The inelastic mean free path is clearly lower than that for elastic collisions, showing that charge transfer is the mechanism that will have the most impact on the IEDF. This has already been reported for helium plasmas [36, 37]. From the values reported in table 2 it can be concluded that, below $10 \mu \mathrm{bar}$, no collisions take place in the sheath and thus, that the kinetic energy of helium ions should equal the potential difference between the plasma and the biased substrate. At $3 \mu$ bar, however, $\lambda_{\text {IMFP }}<L_{s}$ indicating that helium ions experiment in average one charge transfer in the sheath, which could influence the IEDF.

To experimentally check this point, measurements were performed using the simplified retarding field analyzer. The corresponding IEDF are plotted in figure 5 for various helium pressures, for -300 and $-500 \mathrm{~V}$ bias voltages of the grid.
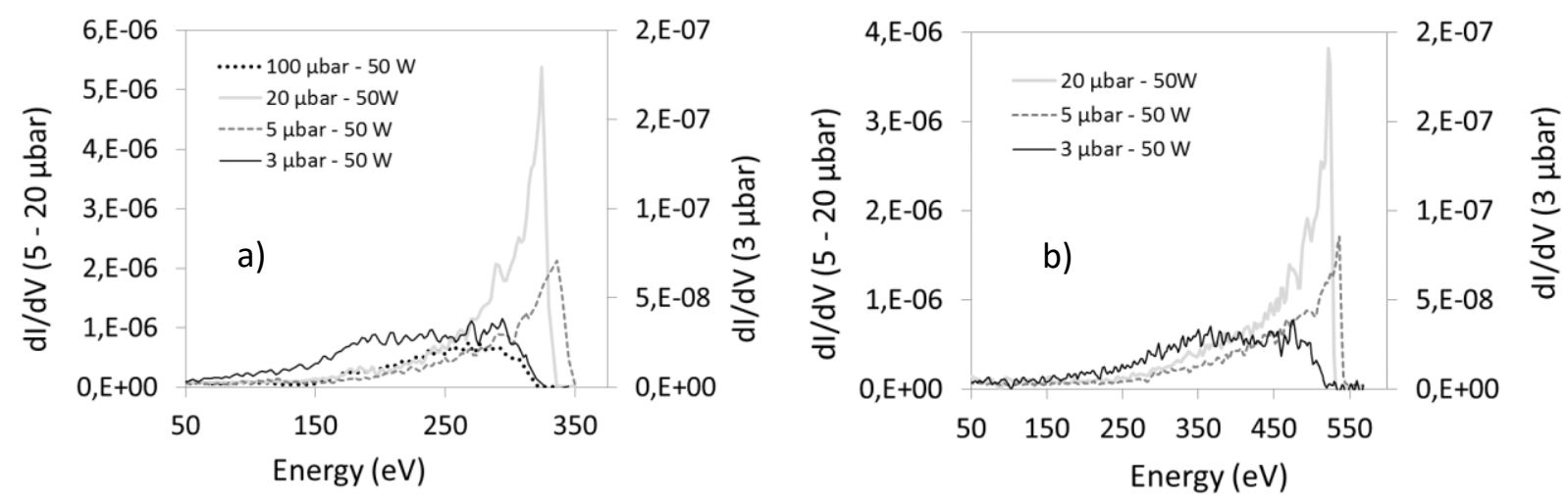

Fig. 5. Ion energy distribution function measured with the home-made simplified retarded field analyzer for a) $-300 \mathrm{~V}$ and b) $-500 \mathrm{~V}$ substrate bias voltages in helium plasma at various pressures and $50 \mathrm{~W}$.

It seems, from these plots that a change in the IEDF occurs between $3 \mu$ bar and $5 \mu$ bar at both bias voltages. A broad distribution is visible at $3 \mu \mathrm{bar}$, whereas the IEDF is narrower and centered at $\left(\mathrm{V}_{\mathrm{p}}-\mathrm{V}_{\mathrm{b}}\right)$ value (maximum energy gained in the sheath) above $5 \mu$ bar. The $\mathrm{V}_{\mathrm{p}}$ values that can roughly be deduced from these graphs are in good agreement with Langmuir probe results. $\mathrm{V}_{\mathrm{p}}$ lies below $20 \mathrm{~V}$ at $3 \mu$ bar and reaches values around $30-40 \mathrm{~V}$ at $5 \mu$ bar and $20 \mu$ bar.

However, it is interesting to note that this change in the IEDF shape coincides with the ion flux jump evidenced in the region (1) of figure 3a. As explained above, the observed phenomena could be due to a modification of the capacitive discharge regime that seems to be $\alpha$ at low pressures, and $\gamma$ at pressures higher than $5 \mu$ bar.

IEDF at the powered electrode in ICP plasmas have been largely studied in the past, and are known to be broad and/or composed of several peaks when capacitive coupling exists; that means in the case of the formation of a large time modulated sheath [26, 37-40]. In literature measurements have proved that a narrow mono-energetic distribution can only be obtained when the capacitive coupling is removed, for instance, by adding an electrostatic shield to the experiment device. These observations have been confirmed by results from RF discharge modeling [26]. The shape of the IEDF has been found to depend upon the number of collisions experimented by the ions accelerated inside the sheath and upon the ion transit time as compared to the RF period [26, 37, 41]. Helium ions being light, their transit time is lower than the RF period for $13.56 \mathrm{MHz}$ which means they will be sensitive to the instantaneous 
sheath potential $[39,41]$. In these conditions a double peak IEDF would be observed in capacitive coupling and a mono-energetic peak in pure inductive one.

Even if the configuration is different from what is reported in literature, since we are dealing with the IEDF at a DC biased substrate, we think that the broad IEDF observed at $3 \mu$ bar, when a faint plasma is formed, can be related to the capacitive nature of the discharge. As evidenced in table 2, at very low pressures, He ions could encounter one charge transfer in the sheath, which would modify the IEDF. In the $\gamma$-CC regime, ionization zone becomes less extended and the IEDF is narrower [27, 28]. As soon as an IC part exists the IEDF exhibits a single peak, as expected.

A closer look to the evolution of the IEDF for pressures above $20 \mu$ bar for $-300 \mathrm{~V}$ shows expected evolution with pressure (see figure 5a): a displacement of the energy peak towards lower values and a broadening. This is due to collisions that helium ions begin to undergo across the accelerating sheath.

Regarding the present work, it is important to note that between $5 \mu$ bar and $20 \mu \mathrm{bar}$, the IEDF is narrow, with a max value corresponding to $\mathrm{V}_{\mathrm{p}}-\mathrm{V}_{\mathrm{b}}$. Below $5 \mu$ bar, the discharge operates in $\alpha$ capacitive mode which seems to result in a broad IEDF distribution. It could be deduced from these observations that, to control the kinetic energy of helium ions, it would be more convenient to carry out implantations in $\gamma$-CC or IC modes. Unfortunately, in our experimental configuration, lowest ion fluxes requested for the planed study have only been reached in $\alpha-C C$. In these conditions implantation occurs with ions carrying a wide range of kinetic energies $\left(150-300 \mathrm{eV}\right.$ for $\left.\mathrm{V}_{\mathrm{b}}=-300 \mathrm{~V}\right)$. However, one has to keep in mind that these energy values lie below the energy threshold for $\mathrm{W}$ atom displacement by He ions [7].

\subsection{He diffusion plasma interaction with the substrate}

As mentioned above, in order to study the effect of low kinetic energy helium ions, implantations have to be carried out in the absence of any other source of energy, or heating. To check this point, measurements have been performed with a dedicated energy flux diagnostic.

Evolution of the energy flux with respect to the pressure is given in figure 6 for $-300 \mathrm{~V}$ bias voltage, and $100 \mathrm{~W}$.

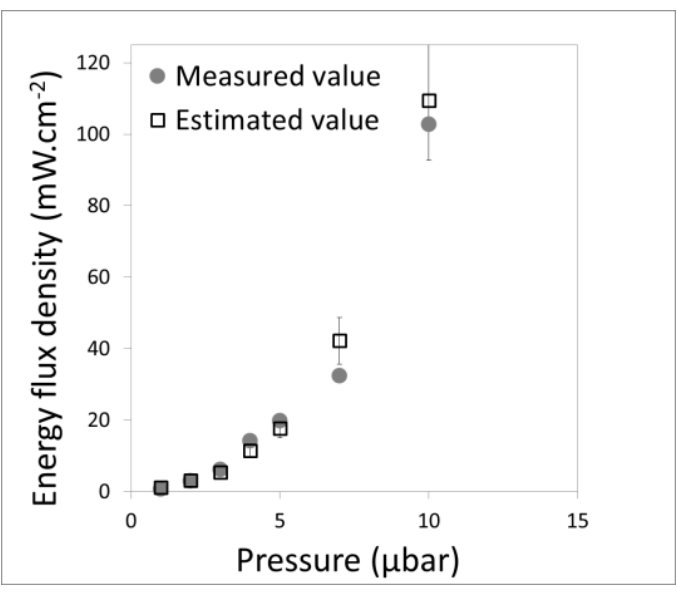


Fig. 6. Energy flux density as measured with the dedicated diagnostic (filled squares) and estimated from the knowledge of the ion flux and assuming an ion kinetic energy equals to the potential difference between the plasma and the biased probe, i.e. maximal energy that ions can gain through the sheath (open squares). $-300 \mathrm{~V}$ bias voltage and $100 \mathrm{~W}$ : region (1) of figure $3 \mathrm{a}$, transition between $\alpha-\mathrm{CC}$ and $\gamma$-CC regimes occurs between 5 and $10 \mu \mathrm{bar}$ (see figure 4). The error bars for the estimated values are estimated from the uncertainty on the measurement of the ionic current.

Measurements are compared to the energy gained by the ions in the sheath estimated using the following formula:

$$
\varphi=F_{i}\left(\left(V_{p}-V_{b}\right)+V_{i}\right) e
$$

Where $\mathrm{F}_{\mathrm{i}}$ is the ionic current measured at the copper and $\mathrm{V}_{\mathrm{i}}$ the ionization potential of helium ions $(24.6 \mathrm{~V})$. In order to take into account at the best the overestimation due to the emission of secondary electrons under ion bombardment, values of the ion secondary electron emission coefficient were roughly estimated from the literature on copper to $0.3,0.21$ and 0.1 for 300 $\mathrm{eV}, 200$ to $250 \mathrm{eV}$ and 100 to $150 \mathrm{eV}$ helium ions, respectively [42].

In this calculation the ions are supposed to carry the maximum energy they can gain across the sheath $\left(\mathrm{e}\left(\mathrm{V}_{\mathrm{b}}-\mathrm{V}_{\mathrm{p}}\right)\right)$, that means a narrow IEDF centered on this value is assumed. The ionization potential is taken into account because it is well known that ions are neutralized during their interaction with a surface, which results in the release of $\mathrm{eE}_{\mathrm{i}}$ energy [43]. Values and trends between measurements and estimations presented in figure 6 are in very good agreement in the entire pressure range. This shows that the energy deposited at the substrate during the implantation experiments is brought by the impinging ions. Even if the IEDF does not exhibit a Dirac peak at $\mathrm{V}_{\mathrm{b}}-\mathrm{V}_{\mathrm{p}}$, especially at low pressures in the $\alpha$-CC regime, the right order of magnitude of the energy influx at the substrate is obtained. Formation of fast neutrals (backscattered ions neutralized at the surface) is often reported in the case of light ions impinging onto heavy elements [44]. These species would contribute to reflect a part of the kinetic energy away from the surface, which would lower the global transferred energy. In our experiment such lowering effect was not observed, indicating that most of helium ions lose their kinetic energy by interaction with the surface.

After considering pressure, the effect of the power injected in the plasma on the deposited energy at the substrate was studied using the energy flux density diagnostic. A typical measurement at $200 \mathrm{~W}$ and $1 \mu$ bar is given in figure 7 . 


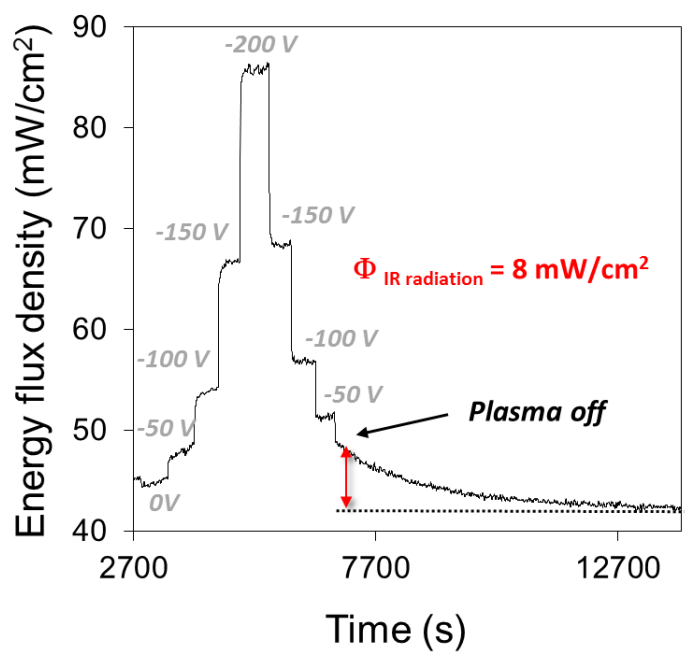

Fig. 7. Energy flux density measurements in helium plasma at $1 \mu$ bar and for $200 \mathrm{~W}$ at the substrate location for increasing (0V to $200 \mathrm{~V}$ ) and decreasing negative bias voltages. After the measurement run a remaining contribution is detected (red arrow) attributed to the IR radiation emitted by the warm plasma source after operating in $\mathrm{CC}$ mode. The dashed horizontal line stands for the offset (energy flux when the plasma is off).

It is seen on figure 7 that a contribution of about $8 \mathrm{~mW} . \mathrm{cm}^{-2}$ with a slow decreasing tail is detected after plasma source operation at $200 \mathrm{~W}$ (in CC mode), when the plasma is lighted off. This behavior is related to a heating process of the plasma source (copper antenna and glass tube) and is due to IR radiations emitted by the hot devices that progressively cool down. This additional energetic contribution is not desired of course, since the aim of the work is to study the effect of helium ion implantation related to their kinetic energy. It has been seen in our study that the IR contribution from the heated source is detected at high RF power in CC mode or, as soon as the discharge operates in IC mode (not shown here). To avoid this, the implantations will be carried out at input RF powers lower than $100 \mathrm{~W}$ and for pressures below $30 \mu$ bar, in CC mode.

\subsection{Implantation conditions}

From the results above ideal implantations conditions have been defined: 1-20 $\mu$ bar, 50-100 $\mathrm{W}$ in $\alpha$ or $\gamma \mathrm{CC}$ regimes and a typical antenna/substrate distance of $35 \mathrm{~cm}$ that can be varied. The size of the antenna could also be modified, we have checked that this would mainly impact the ion flux at the substrate. This ion flux is calculated from the current measured at the substrate, taken into account the secondary emission of electrons under $\mathrm{He}$ ion bombardment.

To vary the number of $\mathrm{He}^{+}$impinging onto the surface, that means the implanted fluence $\left(\mathrm{F}_{\mathrm{i}} \times \mathrm{t}\right)$ at a fixed ion flux, the time should be increased. Consequently the implantation 
conditions should be stable during a long time. Figure 8 shows the time evolution of the energy flux at the substrate in typical implantation conditions for a biased voltage of $-300 \mathrm{~V}$.

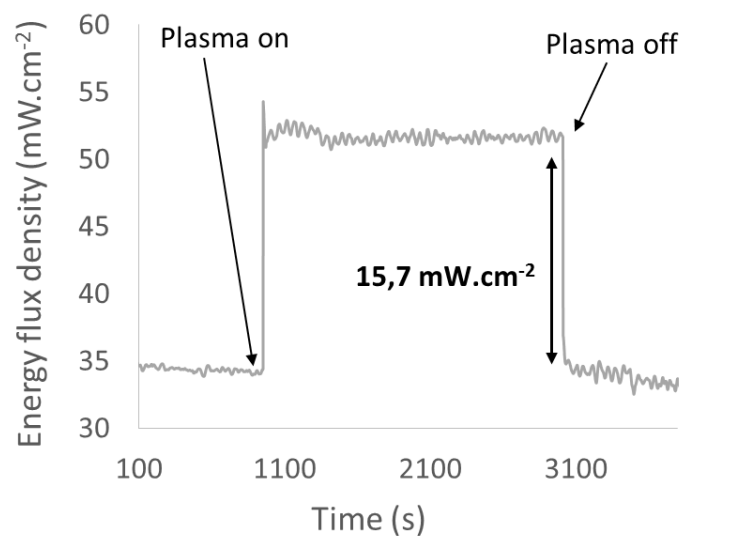

Fig. 8. Energy flux density evolution during a typical implantation experiment at $3 \mu \mathrm{bar}, 100$ $\mathrm{W},-300 \mathrm{~V}$ and during $30 \mathrm{~min}$.

The deposited energy was found very stable which shows that the flux of the incoming ions depositing their kinetic energy is constant. When the plasma is lighted off, the sharp decrease indicates that there is no other energetic contribution than the plasma one. A simple estimation of the mean energy deposited $\left(\mathrm{E}_{\mathrm{dep}}\right)$ by the incoming He ions could be made from the measured energy flux $\varphi$ and ion flux $F_{i}$ :

$$
E_{d e p}=\frac{\varphi}{F_{i}}
$$

It gives the expected value $300 \pm 50 \mathrm{eV}$, showing that the implantation conditions are met to carefully investigate $\mathrm{He}$ insertion effect in the $\mathrm{W}$ lattice.

\subsection{Preliminary results of He implantation in tungsten}

In the present work the effect of two implantation parameters that could be easily varied in the home-made plasma source, i.e. incoming ion flux and total implanted fluence, has been studied by DB-PAS (vacancy defect formation) and NRA (He implanted amount). Chemical analysis of the $\mathrm{W}$ substrates before and after implantation has shown the presence of a thin native oxide. No correlation between the proportion of this oxide and PAS results has been evidenced, showing that oxygen does not induce $\mathrm{W}$ vacancy formation, as expected. In the following, results are thus attributed to the only effect of helium ions.

Implantations have been performed between 20 to $50 \mathrm{~W}$ and for 1 to $3 \mu$ bar helium pressure for a $-300 \mathrm{~V}$ sample bias voltage. The relevant parameters that govern the implantation process are the ion flux and kinetic energy (determined by the discharge regime). Thus, in the following, samples are listed depending on these features. 
In figure 9 the evolution of the $\mathrm{S}$ parameter is given as function of the positron energy for two W samples. Both samples were implanted in conditions leading to similar helium retention fluences at $-300 \mathrm{~V}$ but at different ion flux, meaning in different discharge regimes. For sample 1 implanted at low ion flux $\left(5.7 \times 10^{12}\right.$ ions. $\left.\mathrm{cm}^{-2} \cdot \mathrm{s}^{-1}\right)$ in $\alpha$-CC regime the measured $\mathrm{He}$ fluence is $2.6 \times 10^{15}$ ions.cm ${ }^{-2}$, for sample 2 implanted at higher flux $\left(3.3 \times 10^{14}\right.$ ions. $\left.\mathrm{cm}^{-2} . \mathrm{s}^{-1}\right)$ in $\gamma-\mathrm{CC}$ regime, the measured He fluence is $1.8 \times 10^{15}$ ions. $\mathrm{cm}^{-2}$. In figure 9 the initial and implanted states of both samples are compared. Because the implantation process took place at low kinetic energies, only the first nanometers of the $\mathrm{W}$ are expected to be affected, corresponding to positrons of energy lower than $5 \mathrm{keV}$.

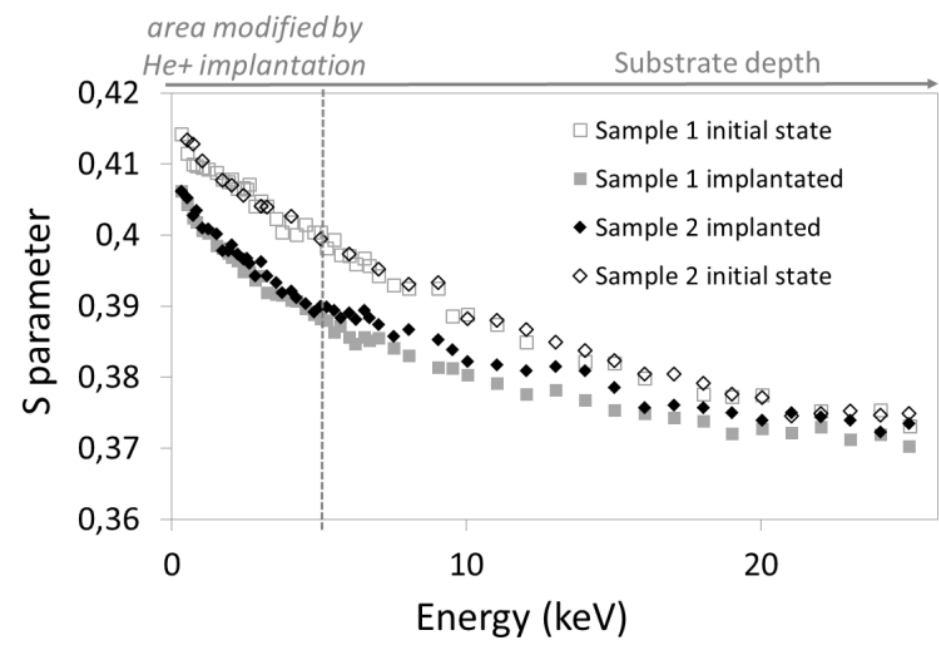

Fig. 9. S annihilation parameter plotted versus the positron energy for two $\mathrm{W}$ samples: open symbols before implantation, filled symbols after implantation. Sample 1 was implanted in $\alpha-$ $\mathrm{CC}$ regime at $5.7 \times 10^{12}$ ions. $\mathrm{cm}^{-2} . \mathrm{s}^{-1}$ sample 2 was implanted in $\gamma$-CC regime at $3.3 \times 10^{14}$ ions. $\mathrm{cm}^{-2} \cdot \mathrm{s}^{-1}$.

The evolution of the $\mathrm{S}$ parameter on the initial states, before implantation, is characteristic of defect free samples. The positron diffusion length calculated from the results is close to 110 $\mathrm{nm}$, indicating that no defect is detected is these samples. This validates the preparation procedure used in this work to remove all vacancies, at least to reduce their concentration below the detection limit of the technique. It is seen that after implantation the $S$ parameter is lower than on the pristine surface, also the positron diffusion length is reduced due to the presence of defects. Starting from a vacancy free surface, a decrease of S could only be due to presence of $\mathrm{W}$ vacancies that are filled with He atoms. This has been previously evidenced on He+ implanted W [8]. It is known that Helium/vacancy complexes can be formed and are very stable [45]. This result points out that vacancies can be formed even when helium ions carry a kinetic energy below $\mathrm{W}$ atom displacement threshold. Another mechanism than kinetic energy transfer has to be considered to explain this result, the so called self-trapping $[46,47]$. The accumulation of helium in the W lattice leads to the formation of He interstitial 
clusters that could induce the displacement of a $\mathrm{W}$ atom, leaving a $\mathrm{W}$ vacancy filled with helium atoms and a W self-interstitial atom.

On figure 9 it is seen that the $S$ annihilation parameter of both samples is the same after implantation, showing that the same implanted state is reached. This result indicates first, that the implantation parameter which plays the main role on the defect formation in $\mathrm{W}$ is the final He retained fluence, rather than the ion flux, even when this last parameter has been varied by a factor-two orders of magnitude. Second, this proves that the discharge regime of the helium plasma has no influence on the modification of $\mathrm{W}$ by $\mathrm{He}^{+}$as detected by DB-PAS technique. This last point strengthens the idea that, below the atom displacement threshold, whatever the ion kinetic energy distribution (a narrow or broad IEDF) in the investigated range, the same elementary mechanisms will take place and thus the effect on $\mathrm{W}$ lattice will be similar.

In figure 10 are displayed the retention fluence measured by NRA as function of the expected fluence $\left(\mathrm{Fi}^{*} \mathrm{t}\right.$ ) (figure $\left.10 \mathrm{a}\right)$ and the $\mathrm{S}$ parameter evolution for samples containing two different amounts of He (figure 10b).
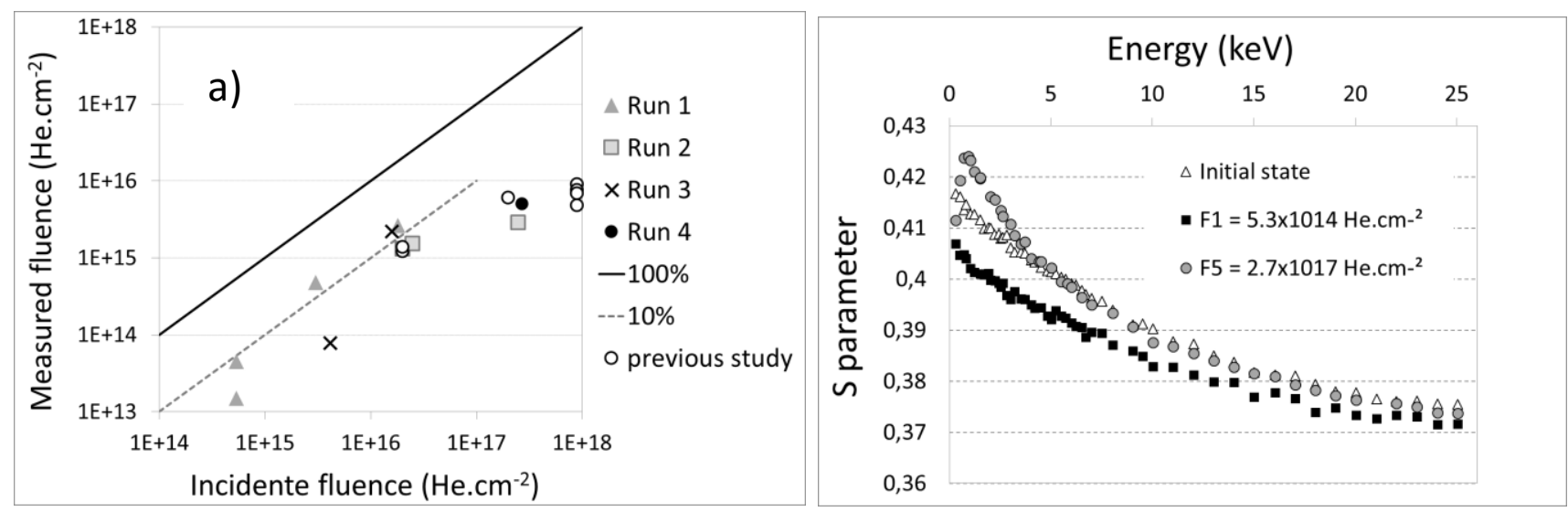

Fig. 10. a) Evolution of the fluence measured by NRA with respect to the incidente fluence $(\mathrm{Fi} * \mathrm{t})$ for various runs of experiments and a previous study [8]. Black line stands for $100 \%$ retention in caption of incident $\mathrm{He}$, dashed line for $10 \%$; b) S parameter evolution with the positron energy for a blank sample, and two implanted samples containing $5.3 \times 10^{14} \mathrm{He} . \mathrm{cm}^{-2}$ and $2.7 \times 10^{17} \mathrm{He} . \mathrm{cm}^{-2}$ respectively.

The evolution observed in figure 10a, i.e. a linear rise on a log-log scale followed by a saturation step, has already been reported by several authors [48-50]. In a previous work this experimental behavior has been compared to molecular dynamics simulation (MDs) of the implantation process, and a very good agreement was found [51]. The very low retention rate of $\mathrm{He}$ in $\mathrm{W}$ (about $10 \%$ in the linear part of the curve) can be related to its very high mobility inside the lattice when not trapped into defects like vacancies. In reference [51], the reason for the saturation step is discussed. It is attributed to the growth of a near-surface dense array of $\mathrm{He}_{\mathrm{n}} \mathrm{V}_{\mathrm{m}}$ complexes (association of $\mathrm{n}$ He atoms trapped in a vacancy where $\mathrm{m} \mathrm{W}$ atoms are missing) preventing the deep penetration of the He ions and promoting the cluster ruptures and the ejection of the He atoms outside the material. In MDs large bubbles have been 
evidenced for high incident fluences. Their coalescence could lead to their rupture through the $\mathrm{W}$ surface, phenomenon occurring when the saturation step is reached.

PAS results presented in figure $10 \mathrm{~b}$ are in good agreement with the above mentioned trends. It is seen that at low fluences, the $S$ parameter value is lower than that of the initial state, again indicating that $\mathrm{W}$ vacancies filled will $\mathrm{He}$ have been formed in the lattice. This supports the fact that in this fluence domain only He trapped in vacancy defect remains stable inside the material. At high fluences, above the saturation step, the $\mathrm{S}$ parameter exhibits a peak at low positron energy, i.e. close to the surface. The rise of the $S$ parameter with the implanted fluence shows that larger free volumes are now present inside the lattice, which could be due to $\mathrm{W}$ vacancy-clusters emptied by the release of previously trapped He atoms. This phenomenon can occur because the implantation takes place very near to the surface. It could also induce local rupture of the $\mathrm{W}$ lattice and thus, formation of porous structures.

All these results should be confirmed by further implantation experiments and simulation and by complementary surface characterization. However, they clearly show the interest and the ability of the home-made plasma source to investigate the implantation of $\mathrm{He}$ in this regime, where accumulation drives the evolution of the metal lattice and of the He retention.

\section{Conclusion}

In this work a plasma implantation source has been designed for $\mathrm{He}^{+}$implantation at low kinetic energies and low ion fluxes. To reach the large requested range of ion fluxes from $10^{11}$ to $10^{14}$ ions.cm ${ }^{-2} \mathrm{~s}^{-1}$, two shapes of antenna (single or 4 turns) were used. It has been shown that, depending on the RF power and the pressure, the discharge operates in either $\alpha-C C, \gamma-$ $\mathrm{CC}$ or IC modes. Despite a DC bias of the substrate, a broad IEDF has been evidenced in the $\alpha$-CC. In $\gamma$-CC or IC modes, the kinetic energy of helium ions is centered close to the expected value when no collisions take place in the accelerating sheath.

Particular attention was paid to precisely control the energetic conditions during implantation. Measurements of the total energy transferred to the substrate have helped choosing the conditions to avoid warming up of the source that would induce indirect heating of the substrate. To fulfill this requirement, low RF power have to be used and the discharge should not operate in IC mode. The home-made plasma source developed in this work appears to be a convenient and versatile implantation source for low energy-low ion flux conditions.

Analysis of the $\mathrm{W}$ samples after implantation has proved that the regime of the CC discharge ( $\alpha$ or $\gamma$ ) has no effect on the final implanted state as qualified by DB-PAS technique. This result indicates that, below the $\mathrm{W}$ atom displacement threshold, a broad or narrow IEDF (in the investigated ranges), will not have a significant effect on the nature of the vacancy defects formed in the material.

Accumulation of He inside the W lattice was found, first, to induce the creation of W vacancies filled with helium. At high fluences, larger free volumes are detected which, in addition to the saturation of the implanted He amount, indicates that part of the incident helium has escaped from its trapping sites. This phenomenon is allowed because the implantation at low kinetic energy takes place very close to the surface. Large vacancy defects 
filled with helium become unstable and can break up, leading to the release of helium and the local rupture of the $\mathrm{W}$ lattice.

\section{Acknowledgement}

The Région Centre Val de Loire and CNRS are acknowledged for financial support. 
References

[1] R. E. Nygren, R. Raffray, D. Whyte, M. Urickson, M. Baldwin, L. L. Snead J. Nucl. Mater. 417 (2011) 451.

[2] J. Roth, E. Tsitrone, A. Loarte, Th. Loarer, G. Counsell, R. Neu, V. Philipps, S. Brezinsek, M. Lehnen, P. Coad, Ch. Grisolia, K. Schmid, K. Krieger, A. Kallenbach, B. Lipschultz, R. Doerner, R. Causey, V. Alimov, W. Shu, O. Ogorodnikova, A. Kirschner, G. Federici, A. Kukushkin J. Nucl. Mater. 390-391 (2009) 1.

[3] W. Kleyn, W. Koppers, N. Lopes Cardozo Vacuum 80 (2006) 1098.

[4] D. Nishijima, M. Y. Ye, N. Ohno, S. Takamura J. Nucl. Mater. 329-333 (1-3 PART B) (2004) 1029.

[5] M. J. Baldwin, R. P. Doerner Nucl. Fusion 48 (2008) 035001.

[6] S. Takamura, T. Miyamoto and N. Ohno Nucl. Fusion 52 (2012) 123001.

[7] K.O.E. Henriksson, K. Nordlund, J. Keinonen, Nucl. Instrum. And Meth. in Phys. Res. B 2006, 244, 377.

[8] P. E. Lhuillier, T. Belhabib, P. Desgardin, B. Courtois, T. Sauvage, M. F. Barthe, A. L. Thomann, P. Brault, Y. Tessier J. Nucl. Mater. 433 (2013) 305.

[9] P.E. Lhuillier, T. Belhabib, P. Desgardin, B. Courtois, T. Sauvage, M.F. Barthe, A.L. Thomann, P. Brault, Y. Tessier Journal of Nuclear Materials 416 (2011) 13.

[10] C. Böhm, J. Perrin Rev. Sci. Instrum. 64 (1993) 31.

[11] A.L. Thomann, N. Semmar, R. Dussart, J. Mathias, V. Lang Review of Scientific Instruments 77 (2006) 033501.

[12] P.A. Cormier, M. Stahl, A.L. Thomann, R. Dussart, M. Wolter, N. Semmar, J. Mathias and H. Kersten J. Phys. D: Appl. Phys. 43 (2010) 465201.

[13] G. Kowarik, M. Brunmayr, and F. Aumayr Nucl. Instruments Methods Phys. Res. Sect. B Beam Interact. with Mater. Atoms 267 (2009) 2634.

[14] D. Gahan, B. Dolinaj, M.B. Hopkins Review of Sci. Instrum. 79 (2008) 033502.

[15] A. Debelle, M. Barthe, T. Sauvage Journal of Nuclear Materials 376 (2008) 216.

[16] V. Sabelová, V. Kršjak, J. Kuriplach, Y. Dai, V. Slugen Journal of Nuclear Materials 458 (2015) 350 .

[17] M. Hakala, M. J. Puska, and R. M. Nieminen Physical Review B 57 (1998) 7621.

[18] T. Sauvage, H. Erramli, S. Guilbert, L. Vincent, M.F. Barthe, P. Desgardin, G.

Blondiaux, C. Corbel, J.P. Piron, F. Labohm, A. Van Veen J. Nucl. Mater. 327 (2004) 159.

[19] F. Paszti Nucl. Instr. and Meth. in Phys. Res. B 66 (1992) 83.

[20] B.M.U. Scherzer, R. Behrisch, J. Roth Nucl. Instr. Meth. 157 (1978) 75.

[21] A. Grill Cold Plasma in Material Fabrication. From Fundamentals to Applications IEE New York (1994) USA.

[22] A. von Engel Plasmas: their nature and Uses Taylor and Francis London (1983) UK. 
[23] J.M. Meek and J.D. Craggs Electrical Breakdown of gases Clarendon Oxford (1953) UK.

[24] I. Peres and M.J. Kushner Plasma Sources Sci. Technol. 5 (1996) 499.

[25] D. Gerst, S. Cuynet, M Cirisan, S. Mazouffre Plasma Sources Sci. Technol. 22 (2013) 015024.

[26] U. Kortshagen Plasma Sources Sci. Technol. 4 (1995) 172.

[27] Se Youn Moon, J. K. Rhee, D. B. Kim, and W. Choe Phys. Plasmas 13 (2006) 033502.

[28] V. A. Godyak, R. B. Piejak, and B. M. Alexandrovich Physical Review Letters 68 (1992) 40.

[29] B. Chapman Glow discharge processes John Wiley \& Sons (1980).

[30] T. E. Sheridan Phys. Plasmas 7 (2000) 3084.

[31] S.-H. Seo, J.I. Hong, K.H. Bai H.Y. Chang Phys. Plasmas 6 (1999) 614.

[32] R. Hegerberg, T. Stefansson and M. T. Elford J. Phys. B: Atom. Molec. Phys. 11 (1978) 133.

[33] C. F. Barnett and P. M. Stier Physical Review 109 (1958) 385.

[34] W. H. Cramer and J. H. Simons The Journal of Chemical Physics 26 (1957) 1272.

[35] S. W. Nagy, W. J. Savola, Jr., and E. Pollack Physical Review 177 (1969) 71.

[36] P.W. May, D. Field, D.F. Klemperer J. Appl. Phys. 71 (1992) 3721.

[37] M.J. Kushner J. Appl. Phys. 58 (1985) 4024.

[38] C. Wild and P. Koidl Appl. Phys. Lett. 54 (1989) 505.

[39] R. J. Hoekstra and M. J. Kushner J. Appl. Phys. 79 (1996) 2275.

[40] U. Kortshagen and M. Zethoff Plasma Sources Sci. Technol. 4 (1995) 541.

[41] E. Kawamura, V. Vahedi, M.A. Lieberman, C.K. Birdsall Plasma Sources Sci. Technol. 8 (1999) R45.

[42] R.A. Baragiola, E.V. Alonso, A.Oliva Florio Physical Review B 19 (1979) 121.

[43] H. Kersten, H. Deutsch, H. Steffen, G.M.W. Kroesen and R. Hippler Vacuum 63 (2001) 385.

[44] T.P. Drüsedau, T. Bock, T.M. John, F. Klabunde and W. Eckstein J. Vac. Sci. Technol. A 17 (1999) 2896

[45] E.V. Kornelsen and A.A. van Gorkum Journal of Nuclear Materials 92 (1980) 79.

[46] J. Thomas, W. A. Swansiger, and M. I. Baskes J. Appl. Phys. 50 (1979) 6942.

[47] W.D. Wilson, C. L. Bisson, M.I. Baskes Phys. Rev. B 24 (1981) 5616.

[48] H.T. Lee, A.A. Haasz, J.W. Davis, R.G. Macaulay-Newcombe, D.G. Whyte, G.M.

Wright J. Nucl. Mater. 363-365 (2007) 898.

[49] A.S. Soltan, R. Vassen, P. Jung J. Appl. Phys. 70 (1991) 793.

[50] J. Amano, D.N. Seidman J. Appl. Phys. 56 (1984) 983. 
[51] L. Pentecoste, P. Brault, A.-L. Thomann, P. Desgardin, T. Lecas, T. Belhabib, M.-F. Barthe, T. Sauvage Journal of Nuclear Materials 470 (2016) 44. 